\title{
Caracterización geobotánica de algunos sintaxones de prados mesófilos (all. Brachypodio-Centaureion nemoralis) del sudoeste de Europa
}

\author{
Gabriel Mercadal i Corominas \\ Herbari de la Universitat de Girona, Facultat de Ciències, Campus Montilivi. C\ M. Aurèlia Campmany, 69. 17003 Girona, \\ Catalunya.
}

\section{Correspondencia}

Gabriel Mercadal i Corominas

e-mail: g.mercadal.corominas@gmail.com

Recibido: 23 febrero 2021

Aceptado: 6 julio 2021

Publicado on-line: 20 julio 2021

Editado por: Andrés V. Pérez Latorre

\begin{abstract}
Resumen
Presentamos sintéticamente una parte de los resultados fitogeográficos descritos en la tesis doctoral del autor. En este caso, caracterizamos y validamos algunos sintaxones de prados mesófilos de la alianza Brachypodio-Centaureion nemoralis (ord. Arrhenatheretalia elatioris, class. Molinio-Arrhenatheretea) del sudoeste de Europa. En total, estudiamos geobotánicamente ocho sintaxones a partir de tablas sintéticas y análisis factoriales de correspondencias publicados previamente en la tesis: una alianza (Brachypodio-Centaureion nemoralis), cinco asociaciones y dos subasociaciones (Campanulo subrhomboidalis-Cynosuretum cristati; Phleo nodosi-Cynosuretum cristati; Cypero longi-Cynosuretum cristati nova; Pediculari schizocalycis-Galietum veri stat. novus; Scorzonero humilisAgrostidetum capillaris nova subass. typicum, subass. serapiadetosum linguae nova).
\end{abstract}

Palabras clave: Europa sudoccidental, revisión sintaxonómica, validación fitosociológica, Brachypodio-Centaureion nemoralis, Arrhenatheretalia elatioris.

\section{Résumé}

Caractérisation géobotanique de certains syntaxons de prairies mésophiles (all. Brachypodio-Centaureion nemoralis) du sud-ouest de l'Europe

\begin{abstract}
Nous présentons synthétiquement une partie des résultats phytogéographiques décrits dans la thèse de doctorat de l'auteur. Dans ce cas, nous caractérisons et validons quelques syntaxons de prairies mésophiles de l'alliance BrachypodioCentaureion nemoralis (ord. Arrhenatheretalia elatioris, class. MolinioArrhenatheretea) du sud-ouest de l'Europe. Au total, nous avons étudié géobotaniquement huit syntaxons à partir de tableaux synthétiques et d'analyses factorielles de correspondance préalablement publiées dans la thèse : une alliance (Brachypodio-Centaureion nemoralis), cinq associations et deux sousassociations (Campanulo subrhomboidalis-Cynosuretum cristati; Phleo nodosiCynosuretum cristati; Cypero longi-Cynosuretum cristati nova; Pediculari schizocalycis-Galietum veri stat. novus; Scorzonero humilis-Agrostidetum capillaris nova subass. typicum, subass. serapiadetosum linguae nova).
\end{abstract}

Mots-clés : Europe du Sud-Ouest, revue syntaxonomique, validation phytosociologique, Brachypodio-Centaureion nemoralis, Arrhenatheretalia elatioris.

\section{Introducción}

En 2019, el autor de este artículo defendió en la Universitat de Girona su tesis doctoral sobre los prados de siega de la región mediterránea de Cataluña (Mercadal, 2019a, 2019b, 2019c). Sin embargo, al tratarse de una monografía, los nuevos sintaxones descritos son inválidos según el artículo 1 del Código Internacional de Nomenclatura Fitosociológica (CINF) (Theurillat et al., 2020). Así pues, en este documento validamos y caracterizamos geobotánicamente algunos sintaxones que incluyen prados de siega mesófilos de la Europa sudoccidental de la alianza Brachypodio-Centaureion nemoralis (ord. Arrhenatheretalia elatioris, class. MolinioArrhenatheretea), del mismo modo que ya hemos realizado para otras unidades sintaxonómicas en Mercadal (2020a, 2020b, 2020c, 2020d, 2021).

\section{Metodología}

Caracterizamos geobotánicamente y validamos fitosociológicamente ocho sintaxones (una alianza, cinco asociaciones y dos subasociaciones) de la alianza Brachypodio-Centaureion nemoralis (ord. Arrhenatheretalia elatioris, class. Molinio-Arrhenatheretea) estudiados, previamente, en la memoria doctoral del autor (Mercadal, 2019c: Tabla 84).

La zona de estudio corresponde al sudoeste europeo, desde Portugal hasta el mediodía de Francia (Figura 1), haciendo especial atención al territorio catalán y sus alrededores. Hemos seguido el método fitosociológico 
tradicional de la escuela sigmatista (Braun-Blanquet, 1964, 1979), así como una línea de clasificación sintética. Los inventarios (Mercadal, 2019c: Tabla 84) los hemos comparado mediante tablas sintéticas y análisis factoriales de correspondencias (AFC). Estos datos analíticos y otros ecológicos y corológicos los referenciamos en el artículo mediante citaciones bibliográficas directas a la tesis. Solo reproducimos tres mapas (Figuras 1-3) y un AFC (Figura 4) que consideramos esenciales para comprender mejor el texto.

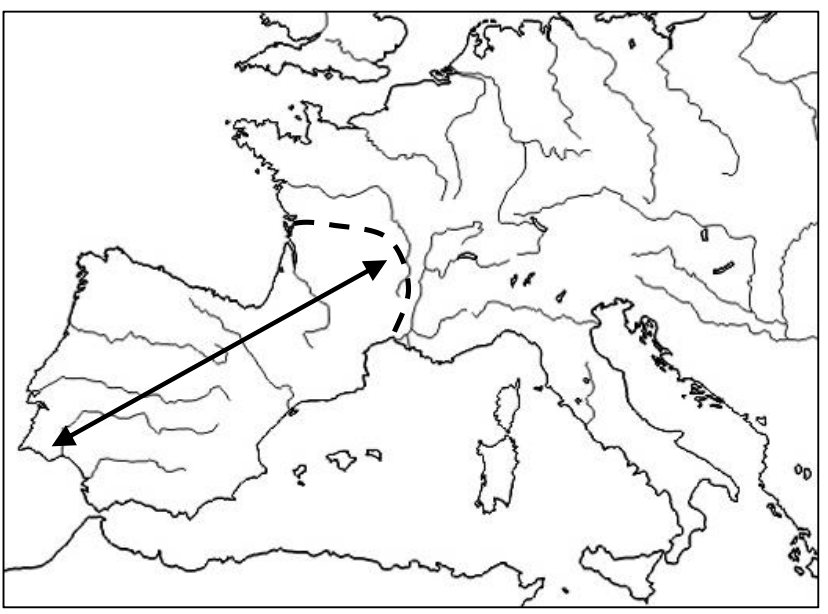

Figura 1. Localización de la zona de estudio en el sudoeste de Europa.

Figure 1. Localisation de la zone d'étude dans le sud-ouest de l'Europe

Las abreviaciones sintaxonómicas utilizadas en latín o inglés son las propuestas por Mucina et al. (2016) o Theurillat et al. (2020). El resto de símbolos y abreviaturas en castellano son: caract., característica/as; dif., especie diferencial; esp., especie; incl., incluye; inv., inventario; p., página; p. ej., por ejemplo; \#, número del sintaxon estudiado.

Respecto a la nomenclatura de los taxones, seguimos la empleada en Mercadal (2019b) que corresponde, básicamente, a la seguida por Bolòs et al. (2005) o, en su defecto, la utilizada por Tison \& Foucault (2014) o Castroviejo (1986-2021). Excepcionalmente, hemos seguido otras obras, las cuales están indicadas en Mercadal (2019b: Tabla 3). Así pues, solo indicamos la autoría de los taxones usados por primera vez en el apartado de especies características (o en su defecto, diagnósticas o diferenciales) de cada sintaxon caracterizado geobotánicamente.

Al final del trabajo incluimos el esquema sintaxonómico de las unidades fitosociológicas estudiadas, así como el nombre completo y la autoría del resto de sintaxones mencionados en el texto.

\section{Resultados y discusión}

Class. Molinio caeruleae-Arrhenatheretea elatioris Tx. 1937 [cf. Mercadal, 2019c: 71, 2020b]

Ord. Arrhenatheretalia elatioris Tx. 1931 [cf. Mercadal, 2019c: 335, 2020d]
1. All. Brachypodio pinnati-Centaureion nemoralis Br.-BI. 1967 [cf. Mercadal, 2019c: 466]

Sinónimos: Brachypodio-Centaureion nemoralis $\mathrm{Br}$-Bl. 1967 (orig. form); Lino biennis-Gaudinion fragilis (Br.-Bl. 1967) de Foucault 1989 nom. superfl. (Art. 29c); Festuco-Brachypodion pinnati Nègre 1969 p. p.; Gaudinio fragilis-Cynosurion cristati (Rivas Goday et Rivas-Martínez 1963) Géhu 1999; Gaudinio fragilisCynosurion cristati (Rivas Goday et Rivas-Martínez 1963) Géhu 2006 nom. inval. (Art. 5); Brachypodio rupestris-Centaureion nemoralis Br.-Bl. 1967 corr. Foucault 2016 nom. inval. (Art. 43, 48c). Pseudónimo: Cynosurion cristati Tx. 1947. Nombres equivalentes: Gaudinio fragilis-Cynosurenion cristati Rivas Goday et Rivas-Martínez 1963 [Gaudinio-Cynosurion Rivas Goday et Rivas-Martínez 1963 (orig. form)]; Lino angustifolii-Oenanthenion pimpinelloidis de Foucault 2016 p. p. nom. inval. (Art. 3m, 24b); Brachypodio rupestris-Gaudinienion fragilis de Foucault 2016 p. p. Otra denominación: Cynosurion cristati Tx. 1947 variante submediterránea con Gaudinia fragilis dominante (Rivas Goday \& Rivas-Martínez, 1963).

Holotypus: Gaudinio fragilis-Festucetum pratensis Br.-BI. 1967 [= Lino angustifolii-Cynosuretum cristati Allorge ex Oberd. et Tx. in Tx. et Oberd. 1958]; designado en Braun-Blanquet (1967: 53) (Art. 18).

Fisionomía y composición florística en el sudoeste de Europa: prados mesófilos, de 50-80(150) cm de altura y con una cobertura del $100 \%$. Las comunidades suelen estar dominadas por hemicriptófitos con valor forrajero: Cynosurus cristatus, Trifolium pratense, $T$. repens y Lolium perenne, aunque florísticamente, se distingue por un conjunto de taxones comunes en suelos de reacción ácida (cf. esp. caract. y dif.) y por distintas plantas típicas de los Arrhenatheretalia (Arrhenatherum elatius s.l., Achillea millefolium aggr., Trisetum flavescens subsp. flavescens, Crepis capillaris, etc.) y de los Molinio-Arrhenatheretea (Dactylis glomerata subsp. glomerata, Taraxacum sect. Taraxacum, Poa trivialis s.l., Prunella vulgaris, Plantago lanceolata, Anthoxanthum odoratum, Lotus corniculatus subsp. corniculatus, Holcus lanatus, Bellis perennis, Rumex acetosa s.l., etc.) (Mercadal, 2019c: Tabla 117 y Anexo 2).

Especies características y diferenciales en el sudoeste de Europa: Agrostis capillaris L. s.I. [incl. A. castellana Boiss. et Reut.], Danthonia decumbens (L.) DC., Potentilla erecta (L.) Raeusch., Carex ovalis Gooden. (dif.), Hieracium pilosella L. (dif.), Myosotis discolor Pers. s.l., Carum verticillatum (L.) W. D. J. Koch (dif.), Centaurea nigra L. s.l. (y formas híbridas o cercanas morfológicamente), Senecio jacobaea L. (dif.), Avenula marginata s.l. (Lowe) Holub [incl. A. sulcata (J. Gay ex Boiss.) Dumort.] (dif.), Nardus stricta L. (dif.), Chamaemelum nobile (L.) All. (dif.), Lepidium heterophyllum Benth. (dif.), Dactylorhiza maculata (L.) Soó s.l. [incl. D. fuchsii (Druce) Soó] (dif.), Colchicum montanum L. [= Merendera montana (L.) Lange, $M$. pyrenaica (Pourr.) P. Fourn.] (dif.), Hypochaeris radicata L. (dif.), Prunella vulgaris $\mathrm{L}$. (dif.). 
Distribución: según nuestros datos actuales, la alianza se extiende por el sudoeste de Europa: cordilleras centrales ibéricas, cordillera cantábrica, Pirineos, llanura aquitana y macizo septentrional occitano (macizo Central francés); pero RodríguezRojo et al. (2017) la hacen llegar todavía hasta las islas británicas y los dominios atlántico y subatlántico del continente europeo, así como en algunos enclaves de la Europa central templada. Por otra parte, Reymann et al. (2016) la indican de Córcega (Figura 2).

Ecología: prados mesófilos, segados y/o pastoreados, instalados en valles fluviales y planicies de los pisos colino, supramediterráneo y montano, principalmente sobre suelos oligotróficos de reacción ácida 0 neutra. Climáticamente, se distribuyen básicamente por los dominios termo-atlántico, de media montaña y del cinturón supramediterráneo establecidos por Ozenda (1994). Los prados se han mantenido desde antiguo mediante las mismas técnicas agropecuarias que los prados del Arrhenatherion elatioris (Mercadal, 2019c: 354, 2020d), pero, en este caso, el pasto suele ser mucho más frecuente y, a menudo, predominante.

Sintaxonomía: tal y como hemos demostrado en Mercadal (2019c: Anexo 2 y Figura 148, 2020d), entre los pastos y los prados de siega mesófilos basales y de media montaña del orden Arrhenatheretalia elatioris de la Europa occidental podemos distinguir florística, ecológica y geográficamente tres alianzas: Arrhenatherion elatioris, de distribución centroeuropea (Mercadal, 2020d), Salvio pratensisDactylidion glomeratae, endémica de la península itálica (Mercadal, 2019c: 351), y BrachypodioCentaureion [\#1], propia del sudoeste europeo.

En la península ibérica, esta interpretación coincide en parte con la propuesta de Rivas-Martínez et al. (2001, 2011), al incluir como sinónimo sintaxonómico de la alianza Cynosurion cristati al Lino-Gaudinion (syn. superfl. del Brachypodio-Centaureion); con la de Géhu (1999), en distinguir una alianza particular (Gaudinio fragilis-Cynosurion cristati) para diferenciar los pastos atlántico-mediterráneos de los centroeuropeos y de los montanos (Cynosurion cristati); y aún más, con la de Rodríguez-Rojo et al. (2017), al incluir todas las asociaciones ibéricas adscritas tradicionalmente al Cynosurion cristati al Brachypodio-Centaureion. En este último caso, como ya hemos mencionado, los autores dan un enfoque geográfico mucho más amplio a la alianza Brachypodio-Centaureion que el propuesto inicialmente por Braun-Blanquet (1967), y le adscriben distintos pastos y prados de siega oligotróficos de las regiones atlántica y submediterránea del continente europeo y de las islas británicas.

Nuestra propuesta para el BrachypodioCentaureion solo incluye, de momento, los prados de nuestra área de estudio, la península ibérica y el sudoeste de Francia, pero esta vez, también le adscribimos algunas asociaciones ibéricas que tradicionalmente han sido asignadas al Arrhenatherion (cf. apartado de variabilidad).
En nuestra interpretación fitosociológica la división entre el Cynosurion cristati y el BrachypodioCentaureion es básicamente florística y geográfica, entendiendo el Brachypodio-Centaureion como a una alianza vicariante, en el sudoeste europeo, de la alianza Cynosurion cristati, que consideramos centroeuropea. Las especies diagnósticas de la alianza Brachypodio-Centaureion propuestas por Rodríguez-Rojo et al. (2017) coinciden con las nuestras, pero nosotros proponemos más taxones para nuestra zona de estudio de menor tamaño (cf. esp. caract. y dif.). En cambio, el Cynosurion cristati de la Europa central se distingue por un conjunto de taxones raros 0 ausentes en el BrachypodioCentaureion: Ranunculus repens, Agrostis stolonifera, Phleum pratense s.l., Plantago major, Lolium perenne, Cardamine pratensis, Scorzonera autumnalis, Alopecurus pratensis subsp. pratensis, Bellis perennis, Poa trivialis s.I., P. annua s.l., Carex nigra, Carex disticha y Potentilla anserina.

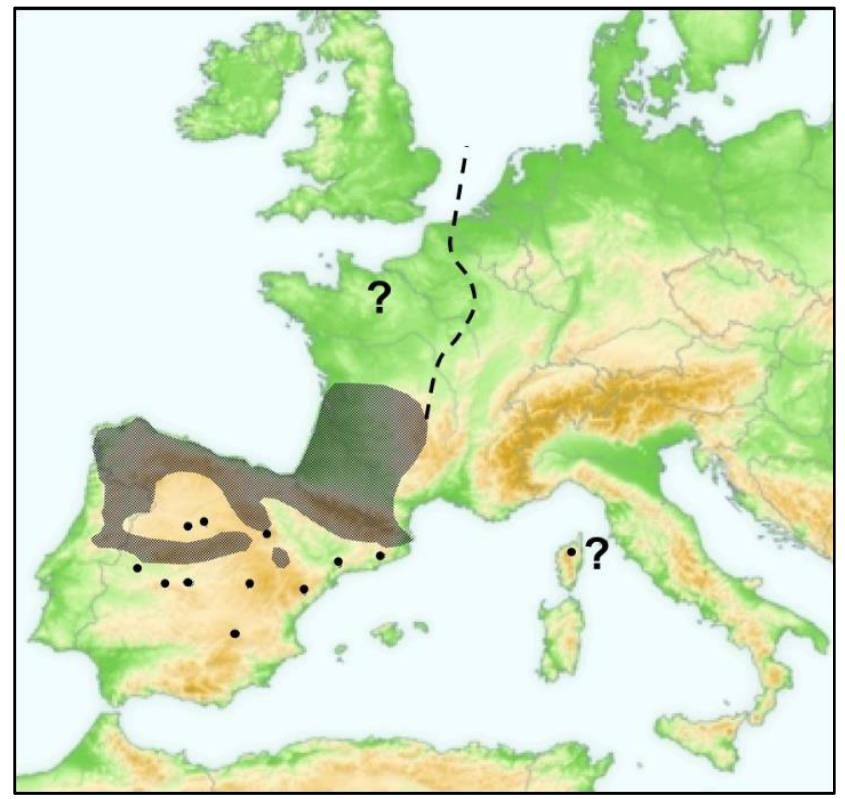

Figura 2. Distribución de la alianza Brachypodio-Centaureion en el sudoeste de Europa. Modificado de Mercadal (2019c: Figura 206).

Figure 2. Répartition de l'alliance Brachypodio-Centaureion dans le sud-ouest de l'Europe. Modifié de Mercadal (2019c : Figure 206).

Respecto a la corrección del nombre propuesta por Foucault (2016) (Brachypodio rupestrisCentaureion nemoralis) y seguida por RodríguezRojo et al. (2017), hay que tener en cuenta que en primer lugar es inválida (Art. 43, 48c), ya que Foucault no indicó explícitamente detrás del nombre corregido la expresión latina "nom. corr. hoc loco". Y, en segundo lugar, según nuestra opinión, esta corrección aún no tiene el fundamento florístico suficiente para ser aplicada. Cabe recordar que Braun-Blanquet (1967) usó Brachypodium pinnatum (L.) P. Beauv. como primer taxon para nombrar la alianza, y según GBIF (2021), WCPV (2021) y el borrador de López (2021) para Flora iberica, en la península solo existe $B$. pinnatum y, además, estos 
autores consideran $B$. rupestre (Host.) Roem. et Schult. como una subentidad de $B$. pinnatum con poco valor taxonómico. Por lo tanto, López desestima explícitamente la opinión de Schippman (1991), el monógrafo del género Brachypodium que considera que en el sudoeste de Europa solo existe $B$. rupestre, así como la opinión más reciente de Tison \& Foucault (2014) y de Pignatti et al. (2017) que siguen el criterio del botánico alemán.

Por otro lado, por lo que se refiere al segundo nombre de la alianza, Centaureion nemoralis, según Gardou (1972) y Arnelas \& Devesa (2011) en Euskadi, el área de estudio donde Braun-Blanquet (1967) describió el Brachypodio-Centaureion, no se desarrolla Centaurea nigra L. subsp. nemoralis (Jordan) Gremlin [= C. nemoralis Jordan]. Según Arnelas \& Devesa (2011) las centaureas adscritas a $C$. nemoralis en la península ibérica corresponden a $C$. nigra $\mathrm{L}$. subsp. nigra. Así pues, en principio, si ambas centaureas correspondiesen a dos especies distintas habría que corregir el nombre Brachypodio-Centaureion nemoralis por Brachypodio-Centaureion nigrae (Art. 43). Sin embargo, como todavía no tenemos nada claro el valor taxonómico de $C$. nemoralis, ni su jerarquía, ni su posible presencia en los prados estudiados por BraunBlanquet, ni tan siquiera qué entidad en concreto observó Braun-Blanquet (podría ser $C$. debeauxii Godr. et Gren. in Gren. et Godr. o alguna entidad del grupo $C$. decipiens Thuill.), de momento preferimos mantener intacto todo el nombre original de la alianza siguiendo las recomendaciones del CINF.

En cuanto a la asociación Gaudinio-Festucetum, el tipo nomenclatural de la alianza BrachypodioCentaureion, según nuestra opinión corresponde a un sinónimo sintaxonómico posterior del LinoCynosuretum. El tipo del Gaudinio-Festucetum ha sido designado recientemente por Rodríguez-Rojo et al. (2017: 716): Braun-Blanquet (1967), Tabla 19, inv. 12.

Finalmente, respecto algunos aspectos sobre la sinonimia de la alianza, por un lado, Géhu (1999: 19) promocionó a alianza a la suball. Gaudinio fragilisCynosurenion cristati descrita por los Rivas, escogiendo como holotipo el Lino biennisCynosuretum cristati (orig. form) (Art. 18a); pero, posteriormente, Géhu (2006: 63) volvió a indicar, por error y sin holotipo, el Gaudinio-Cynosurion como un status novus. Por este motivo, Mucina et al. (2016: 62) indican como inválida la alianza del 2006 (Art. 5).

Por otro lado, recientemente, Foucault (2016) ha descrito dos subalianzas ecológicas dentro del Brachypodio-Centaureion: el Lino angustifoliiOenanthenion pimpinelloidis, para las asociaciones mesohigrófilas, y el Brachypodio rupestrisGaudinienion fragilis, para las mesófilas. Sin embargo, el Lino-Oenanthenion es inválido, ya que Foucault le adscribe el tipo nomenclatural del Brachypodio-Centaureion, la asociación GaudinioFestucetum, como si fuera la subalianza típica, pero el nombre del Lino-Oenanthenion no corresponde al nombre de la alianza con la desinencia de subalianza, tal como especificaba la tercera edición el CINF (Art. $3 \mathrm{~m}, 24 \mathrm{~b})$ de Weber et al. (2000). Asimismo, según el código actual (Theurillat et al., 2020), la subalianza típica correspondería simplemente al autónimo EuBrachypodio-Centaurenion (Art. 24b).

Variabilidad: de momento, en el sudoeste europeo incluimos a 14 asociaciones (Mercadal 2019c: Tabla 84), de las cuales solo estudiamos cinco por ser nuevas o desarrollarse en Cataluña [cf. \#], el área central de la tesis: Agrostio castellanae-Cynosuretum cristati, Caro verticillati-Cynosuretum cristati, Lino angustifolii-Cynosuretum cristati [incl. GaudinioFestucetum pratensis], Colchico montaniCynosuretum cristati nom. corr., Campanulo subrhomboidalis-Cynosuretum cristati nom. inept. [\#1.1], Agrostio castellanae-Arrhenatheretum bulbosi, Anthemido nobilis-Cynosuretum cristati, Festuco amplae-Cynosuretum cristati, Orchido morionisSerapiadetum linguae, Armerio segoviensisArrhenatheretum bulbosi, Phleo nodosi-Cynosuretum cristati [\#1.2], Cypero longi-Cynosuretum cristati nova [\#1.3], Pediculari schizocalycis-Galietum veri stat. novus [\#1.4], Scorzonero humilis-Agrostidetum capillaris nova [\#1.5].

No corregimos el nombre de la asociación Orchido morionis-Serapiadetum linguae, ya que aún no está claro el nombre más apropiado para Orchis morio. Algunos autores consideran que el nombre correcto es Orchis morio L. (Aedo \& Herrero, 2005; Bolòs et al., 2005; Kaplan et al., 2019), otros Anacamptis morio (L.) R. M. Bateman, Pridgeon et M. W. Chase (Bateman et al., 1997; Tison \& Foucault, 2014; Pignatti et al., 2017; Parolly \& Rohwer, 2019; GBIF, 2021; WCPV, 2021), y aún, algunos pocos, Herorchis morio (L.) D. Tyteca et E. Klein (Tyteca \& Klein, 2008; Delforge, 2009).

Respecto al Merendero pyrenaicae-Cynosuretum cristati, esta vez, aunque algunos autores consideran que el nombre correcto de Merendera pyrenaica es M. montana (Aizpuru et al., 1999; Bolòs et al., 2005; Rico, 2013a) y otros Colchicum montanum (Fennane et al., 2014; Tison \& Foucault, 2014; GBIF, 2021; WCPV, 2021), corregimos el nombre de la asociación por el de Colchico montani-Cynosuretum cristati, ya que los estudios filogenéticos más recientes avalan la integración del género Merendera dentro del género Colchicum (Rico, 2013b: 86).

\subsection{Ass. Campanulo subrhomboidalis-Cynosuretum} cristati Nègre 1969 nom. inept. (Art. 44) [cf. Mercadal, 2019c: 478]

Sinónimos: Campanulo-Cynosuretum cristati Nègre 1969 (orig. form); Association à Cynosurus cristatus et Campanula subromboidalis Nègre 1969 (orig. form); Cynosuretum catalaunicum Vigo 1968 prov. nom. inval. (Art. 3b); Cynosuro cristati-Trifolietum repentis O. Bolòs (1967) 1983 p. p. nom. illeg. (Art. 31); Carici ornithopodae-Agrostidetum capillaris Villegas 1997 (syntax. syn. prov.).

Especies diagnósticas: Agrostis capillaris, Festuca rubra L. s.I., Achillea millefolium L. aggr., Cerastium fontanum Baumg. s.l., Prunella vulgaris, Alchemilla hybrida (L.) L. s.l., Phleum pratense L. subsp. pratense, Pimpinella saxifraga L., Rhinanthus pumilus (Sterneck) Pau, Stachys officinalis (L.) Trevis. (Mercadal, 2019c: Anexo 2 y Tabla 118). 
Lectotypus: Nègre (1969): p. 111, Tabla 29, inv. 442; designado en Foucault (2016: 174).

Ecología y distribución: pastos, a veces segados, del Pirineo y de las cordilleras litorales catalanovalencianas, de (300)800-1.500(1.700) m de altitud (Figura 3).

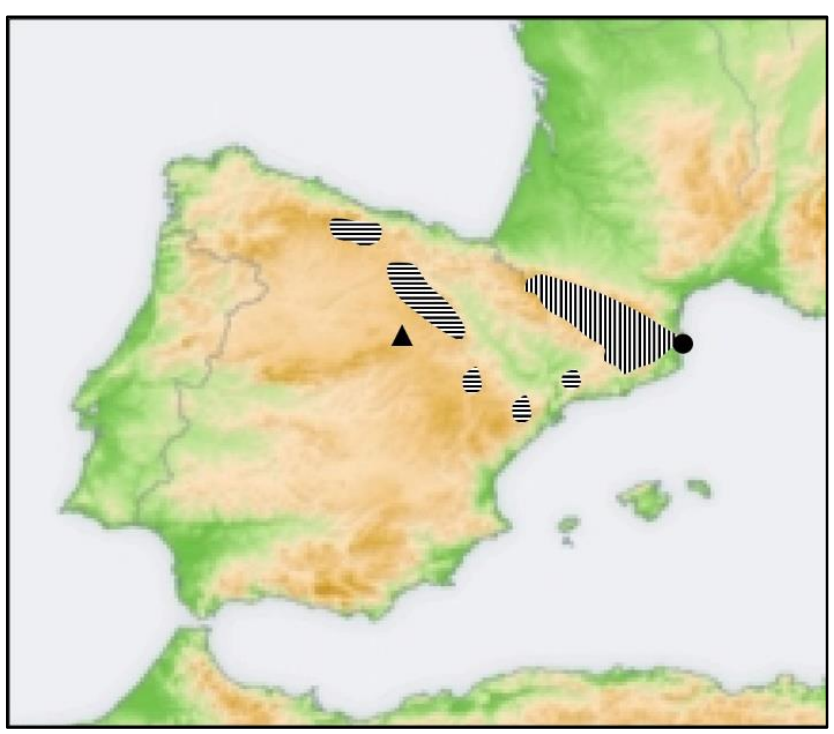

Figura 3. Distribución de las asociaciones CampanuloCynosuretum (rayas verticales), Cypero-Cynosuretum (punto negro), Pediculari-Galietum (triangulo negro) y Phleo-Cynosuretum (rayas horizontales) en la península ibérica. Modificado de Mercadal (2019c: Figura 209).

Figure 3. Répartition des associations CampanuloCynosuretum (rayures verticales), Cypero-Cynosuretum (point noir), Pediculari-Galietum (triangle noir) et PhleoCynosuretum (rayures horizontales) dans la péninsule ibérique. Modifié de Mercadal (2019c : Figure 209).

Sintaxonomía: incluimos dentro de esta asociación a los pastos dominados por Cynosurus cristatus de los Pirineos y de las sierras litorales del norte de la península ibérica, hasta el Montseny y el Cap de Creus (Cataluña), tal como han sugerido previamente Rodríguez-Rojo \& Fernández-González (2014) y Rodríguez-Rojo et al. (2017), y así como nos sugieren nuestros propios resultados (Mercadal, 2019c: Tabla 118).

Para corroborar la separación de las asociaciones dominadas por Cynosurus cristatus en la mitad oriental de la península ibérica, hemos realizado un AFC (Figura 4). En el gráfico, los inventarios pirenaicos y los de las sierras prelitorales del norte de Cataluña se agrupan (Campanulo-Cynosuretum), y quedan claramente separados de los del centro de la península ibérica y de los de las cordilleras litorales del sur de Cataluña y del País Valenciano (Phleo-Cynosuretum), así como de los pastos ligeramente salobres de la península de Cap de Creus (NE de Cataluña) (Cypero-Cynosuretum).

Por otra parte, tendríamos de corregir el nombre de la asociación según el artículo 44 del CINF, ya que Campanula lanceolata Lapeyr. var. subrhomboidalis Nègre 1969, el taxon elegido por Nègre para dar el primer nombre a la asociación es ilegítimo (Geslot, 1982) y no tiene ningún valor taxonómico (L. Sáez y J.-M. Tison, com. pers.). C. lanceolata. var. subrhomboidalis fue descrita por Nègre (1969: 107-
110), y el tipo conservado en el herbario personal de Alain Geslot (G 292). Según Geslot (1982), la variedad de Nègre es un sinónimo posterior de Campanula rotundifolia L. var. major DC. 1839, y, de hecho, según su clave de los taxones infraespecíficos de $C$. rotundifolia, la campánula descrita por Nègre corresponde a C. rotundifolia var. major, ya que presenta un aspecto robusto, tallos floríferos verdes finamente ciliados en su parte inferior y flores grandes ( $>$ de $20 \mathrm{~mm}$ de longitud). Además, según los datos de Geslot et al. (1990: 158), en Banhèras de Luishon (Bagnères-de-Luchon) y sus alrededores de la vertiente norte de los Pirineos centrales (el locus classicus del taxon), solo se encuentra una campánula compatible con la descripción de Nègre (1969): C. rotundifolia s. str. Aun así, J.-M. Tison (com. pers.) nos ha hecho notar que Nègre comenta que las plantas de la var. subrhomboidalis presentan el tallo y las hojas cubiertas de pelos, y que la subsp. rotundifolia, aunque es morfológicamente muy variable, tiene un carácter constante y muy distintivo que es ser siempre glabra (carácter que se confirma en la descripción de la subespecie rotundifolia en Saéz \& Aldasoro (2001: 111) de Flora iberica). Por ese motivo, según Tison, la var. subrhomboidalis se aproxima más a $C$. rotundifolia subsp. hispanica (Willk. in Willk. et Lange) O. Bolòs et Vigo $[=C$. hispanica Willk. in Willk. et Lange] que a $C$. rotundifolia s. str.

Desafortunadamente, no hemos podido consultar el testimonio $\mathrm{G} 292$ a pesar de la búsqueda incesante de la Dra. Isabelle Chanaron en el herbario del Muséum d'histoire naturelle d'Aix-en-Provence. Así pues, de momento, para poder esclarecer esta cuestión taxonómica, es preciso colectar en los lugares donde Nègre (1969) levantó sus tres inventarios del Campanulo-Cynosuretum.

Variabilidad: dentro de esta asociación hemos incluido, provisionalmente, el Carici-Agrostidetum, un sintaxon que se diferencia bien fisionómicamente del tipo de la asociación Campanulo-Cynosuretum por la abundancia de Pteridium aquilinum. En el AFC (Figura 4), los inventarios del Carici-Agrostidetum quedan ligeramente separados del resto de inventarios del Campanulo-Cynosuretum; pero a pesar de ello, florísticamente ambos sintaxones son bastante similares (cf. Villegas, 1997: Tabla 1), y por este motivo entendemos, de momento, el CariciAgrostidetum como una subasociación del Campanulo-Cynosuretum: una comunidad marginal de los alrededores de los hayedos, que solo se mantiene por un pastoreo irregular, y que corresponde a un estadio de sucesión ecológica poco avanzado del Campanulo-Cynosuretum hacia el bosque potencial (esp. dif.: Pteridium aquilinum (L.) Khun, Carex ornithopoda Willd., Satureja vulgaris (L.) Fritsch subsp. vulgaris).

Por otro lado, Villegas (1993) también distingue dentro del Cynosuro-Trifoliteum dos subasociaciones aún inéditas [subass. plantaginetosum mediae Villegas 1993 nom. ined. (Art. 1) y subass. trisetetosum flavescentis Villegas 1993 nom. ined. (Art. 1)] que todavía tenemos que valorar. 
Finalmente, los prados del piso supramediterráneo situados por debajo de los $800 \mathrm{~m}$ de altitud adscritos tradicionalmente al Cynosuro-Trifolietum, los consideramos, a la espera de un estudio más detallado, como fragmentos de asociación.

En definitiva, cabe levantar más inventarios de esta comunidad, especialmente, en la vertiente norte pirenaica y en diferentes regiones por debajo de los $800 \mathrm{~m}$ de altitud, para poder discriminar correctamente sus distintas subasociaciones ecológicas y/o geográficas, así como su distribución general.

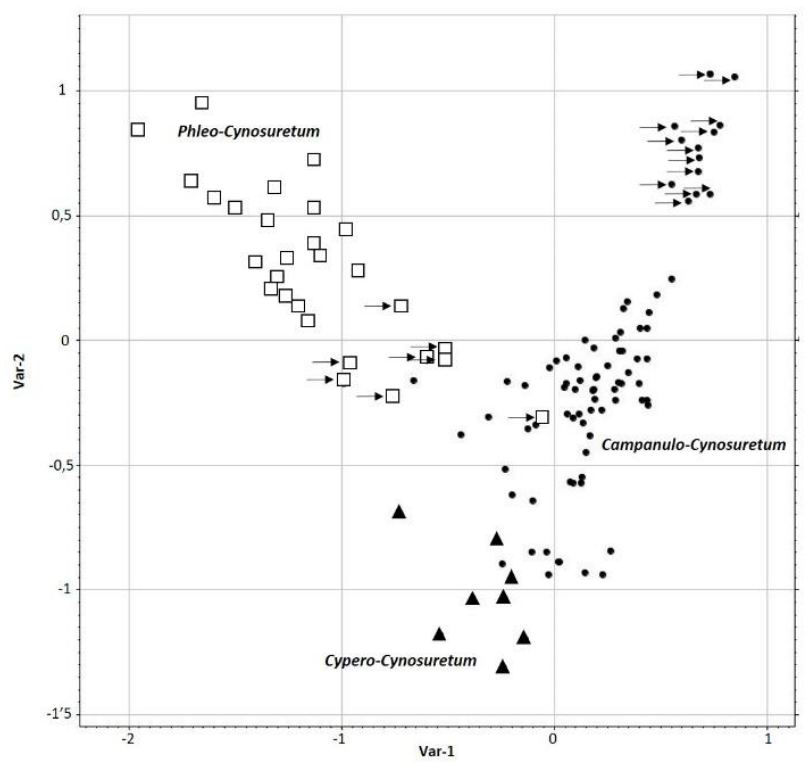

Figura 4. AFC de las asociaciones CampanuloCynosuretum $(\bullet[\rightarrow \bullet$, Carici-Agrostidetum $])$, CyperoCynosuretum (A) y Phleo-Cynosuretum $(\square[\rightarrow \square$, inv. de Cataluña (Bolòs, 1967; Molero, 1976; Álvarez de la Campa, 2004)]). Se representan los dos primeros ejes, que acumulan una varianza conjunta del $13,7 \%$ (Mercadal, 2019c: Figura 208).

Figure 4. AFC des associations Campanulo-Cynosuretum $(\boldsymbol{\bullet}[\rightarrow$, Carici-Agrostidetum]), Cypero-Cynosuretum $(\mathbf{A})$ et Phleo-Cynosuretum ( $\square[\rightarrow \square$, inv. de Catalogne (Bolòs, 1967; Molero, 1976; Álvarez de la Campa, 2004)]). Les deux premiers axes sont représentés, qui cumulent une variance combinée de 13,7\% (Mercadal, 2019c : Figure 208).

1.2. Ass. Phleo nodosi-Cynosuretum cristati Rodríguez-Rojo et Fernández-González 2014 [cf. Mercadal, 2019c: 478]

Sinónimos: Cynosuretum pradense Bolòs 1967 nom. illeg. (Art. 34); Cynosuro cristati-Trifolietum repentis $\mathrm{O}$. Bolòs (1967) 1983 p. p. nom. illeg. (Art. 31).

Especies diagnósticas: Phleum pratense L. subsp. nodosum (L.) Dumort., Bellis perennis L., Festuca trichophylla (Ducros ex Gaudin) K. Richt., Hieracium pilosella, Agrostis capillaris L. subsp. castellana (Boiss. et Reut.) O. Bolòs, Masalles et Vigo, Trifolium striatum L., Deschampsia cespitosa (L.) P. Beauv. (Mercadal, 2019c: Anexo 2 y Tabla 118).

Holotypus: Rodríguez-Rojo \& Fernández González (2014), p. 477, Tabla 3, inv. 1.
Ecología y distribución: pastos, a veces segados, del sistema lbérico y de las cordilleras litorales del sur de Cataluña y del norte del País Valenciano (Figura 3).

Sintaxonomía: agrupamos en una misma asociación (Phleo-Cynosuretum) los prados del orden Arrhenatheretalia del sur de Cataluña y del norte del País Valenciano, tal como han propuesto anteriormente Rodríguez-Rojo \& Fernández-González (2014) y Rodríguez-Rojo et al. (2017). Hasta entonces, estos prados catalanes dominados por Cynosurus cristatus habían sido adscritos al Cynosuretum pradense, un nombre ilegítimo (Art. 34) por utilizar un término ecológico; o bien, al Cynosurion-Trifolietum, que también es ilegítimo (Art. 31), porque corresponde a un homónimo posterior del Cynosuro cristatiTrifolietum repentis Cortini-Pedrotti et al. 1973.

Variabilidad: no hemos realizado ningún estudio específico para poder diferenciar distintos agrupamientos vegetales dentro de esta asociación. Hacen falta más inventarios del interior de la península $y$, sobre todo, de las tierras catalanas. En el AFC (Figura 4) se puede ver claramente que los prados catalanes (Bolòs, 1967; Molero, 1976; Álvarez de la Campa, 2004) son distintos florísticamente de los del centro de la península: en la gráfica los prados de Cataluña quedan situados en una posición intermedia entre el Phleo-Cynosuretum del sistema Central Ibérico y el Campanulo-Cynosuretum pirenaico. La recogida de más datos y su posterior análisis conducirá, seguramente, al establecimiento de distintas subasociaciones geográficas.

1.3. Ass. Cypero Iongi-Cynosuretum cristati Mercadal ass. nova hoc loco [cf. Mercadal, 2019c: 480]

Sinónimos: Cynosuro-Trifolietum repentis sensu Franquesa 1995, non O. Bolòs (1967) 1983 p. p. nom. illeg. (Art. 31); Cypero longi-Cynosuretum cristati Mercadal 2019 nom. ined. (Art. 1).

Holotypus hoc loco designatus: Franquesa (1995), p. 345, Tabla, 26, inv. 5 [sub Cynosuro-Trifolietum repentis]

Especies diagnósticas: Festuca arundinacea Schreb., Cyperus longus L., Plantago crassifolia Forssk., Vicia sativa L. subsp. nigra Ehrh. (probablemente, V. segetalis Thuill.), Trifolium squamosum L., Oenanthe lachenalii C. C. Gmel. (Mercadal, 2019c: Anexo 2 y Tabla 118).

Ecología y distribución: pastos húmedos de Cap de Creus (Alt Empordà, NE de Cataluña), sobre suelos débilmente salobres, de 50-125 m de altitud (Figura 3). Posiblemente, también se extiende más hacia el norte por la costa de la Albera.

Sintaxonomía: los inventarios dominados por Cynosurus cristatus de Cap de Creus habían sido incluidos en la asociación Cynosuro-Trifolietum por Franquesa (1995). Nosotros diferenciamos los inventarios más basales, los que se encuentran encima de suelos ligeramente salinizados por las gotitas transportadas por el viento, y los adscribimos a la nueva asociación Cypero-Cynosuretum (Figura 4). 
En cambio, los prados que no se encuentran influenciados por la sal del mar, situados entre 300 y $500 \mathrm{~m}$ de altitud, corresponden, según nuestra opinión actual, a formas fragmentarias del CampanuloCynosuretum (cf. p. ej. Franquesa, 1995: Tabla 26, invs. 3 y 9 ).

1.4. Ass. Pediculari schizocalycis-Galietum veri (M. Mayor, T. E. Díaz, F. Navarro, F. Martínez et G. Andrés 1975) Mercadal stat. novus hoc loco [cf. Mercadal, 2019c: 483]

Sinónimo: Galio veri-Arrhenatheretum bulbosi sensu Mayor et al. 1975, non (Rivas Goday et Borja 1961) Rivas-Martínez 2002; Pediculari schizocalycisGalietum veri (M. Mayor et al. 1975) Mercadal 2019 nom. ined. (Art. 1). Nombres equivalentes: Galio veri-Arrhenatheretum bulbosi (Rivas Goday et Borja 1961) Rivas-Martínez 2002 subass. nardetosum strictae M. Mayor, T. E. Díaz, F. Navarro, F. Martínez et G. Andrés 1975 [Galio-Arrhenatheretum gudaricum Rivas Goday et Borja 1961 subass. nardetosum Mayor et al. 1975 (orig. form)].

Holotypus hoc loco designatus: Mayor et al. (1975), p. 306, inv. 22 [sub Galio-Arrhenatheretum gudaricum Rivas Goday et Borja 1961 subass. nardetosum Mayor et al. 1975].

Especies diagnósticas: Armeria arenaria (Pers.) Schult. subsp. segoviensis (Gand. ex Bernis) Nieto Fel., Dianthus deltoides L., Pedicularis schizocalyx (Lange) Steininger, Klasea nudicaulis (L.) Fourr., Erodium carvifolium Boiss. et Reut., Juncus squarrosus L., Alopecurus pratensis L. subsp. arundinaceus (Poiret) Husnot (Mercadal, 2019c: Anexo 2).

Ecología y distribución: prados de siega habitualmente pastados sobre terrenos oligrotróficos de reacción ácida o neutra del sector oriental del sistema Central lbérico (Mayor et al., 1975) (Figura 3).

Sintaxonomía y variabilidad: agrupamos los inventarios adscritos inicialmente por Mayor et al. (1975) a tres subasociaciones distintas del GalioArrhenatheretum (typicum, molinietosum Rivas Goday et Borja 1961 y nardetosum Mayor et al. 1975) en una nueva asociación: Pediculari-Galietum (cf. Mercadal, 2019c: Anexo 2 y Figura 163). En estudios posteriores, podremos identificar las posibles subasociaciones ecológicas dentro de la asociación.

\subsection{Ass. Scorzonero humilis-Agrostidetum capillaris Billy ex Mercadal ass. nova hoc loco [cf. Mercadal, 2019c: 481]}

Sinónimos: Scorzonero humilis-Agrostidetum capillaris Billy 2000 prov. nom. inval. (Art. 3b); Scorzonero humilis-Serapietum linguae Billy 2000 prov. nom. inval. (Art. 3b) (orig. form); Scorzonero humilis-Agrostidetum capillaris Billy ex Mercadal 2019 nom. ined. (Art. 1).

Holotypus hoc loco designatus: Billy (2000), p. 220, Tabla VI, inv. D13 [sub Scorzonero humilisAgrostidetum capillaris Billy 2000].
Especies características y diferenciales: Scorzonera humilis L., Carex panicea L. (dif.), Dactylorhiza maculata, Carum verticillatum (dif.), Myosotis nemorosa Besser, Cardamine pratensis L. (dif.) (Mercadal, 2019c: Anexo 2 y Tabla 119).

Ecología y distribución: prados de siega mesohigrófilos, frescos y acidófilos del macizo Septentrional occitano (Auvernia), situados entre los 350 y los $840 \mathrm{~m}$ de altitud (Billy, 2000).

Sintaxonomía: Billy (2000) propuso provisionalmente dos asociaciones distintas (Scorzonero-Agrostidetum y Scorzonero-Serapiadetum) inválidas (Art. 3b), y las adscribió a la alianza Cynosurion cristati. Nosotros hemos creído más conveniente unirlas en una sola asociación nueva y crear dos subasociaciones ecológicas. En cuanto a su adscripción fitosociológica, incluimos provisionalmente la asociación en el Brachypodio-Centaureion. Sin embargo, su asignación es compleja, ya que presenta taxones típicos de diferentes alianzas (Brachypodio-Centaureion, Cynosurion cristati, Calthion palustris y Juncion actutiflori) pero, al mismo tiempo, esta mezcla le confiere una composición florística muy particular y característica.

Variabilidad: distinguimos dos subasociaciones ecológicas: subass. typicum [\# 1.5.1] y subass. serapiadetosum linguae nova [\# 1.5.2].

1.5.1. Ass. Scorzonero-Agrostidetum subass. typicum [cf. Mercadal, 2019c: 481]

Nombre equivalente: Scorzonero humilisAgrostidetum capillaris Billy 2000 prov. nom. inval. (Art. 3b).

Especies diferenciales: Lychnis flos-cuculi L., Stellaria graminea L., Cerastium fontanum subsp. vulgare (Hartman) Greuter et Burdet, Alopecurus pratensis L. subsp. pratensis, Bromus racemosus L., Cirsium palustre (L.) Scop. (Mercadal, 2019c: Tabla 119).

Ecología: prados mesohigrófilos poco intervenidos.

Sintaxonomía: subasociación típica que corresponde a la forma más higrófila de la asociación. Es la más próxima florísticamente a la alianza Cynosurion cristati.

1.5.2. Ass. Scorzonero-Agrostidetum subass. serapiadetosum linguae Billy ex Mercadal subass. nova hoc loco [cf. Mercadal, 2019c: 482]

Sinónimo: Scorzonero-Agrostidetum Billy ex Mercadal 2019 nom. ined. (Art. 1) subass. serapietosum linguae Billy ex Mercadal 2019 nom. ined. (Art. 1) (orig. form). Nombre equivalente: Scorzonero humilis-Serapietum linguae Billy 2000 prov. nom. inval. (Art. 3b) (orig. form).

Holotypus hoc loco designatus: Billy (2000), p. 220, Tabla VI, inv. L664 [sub Scorzonero humilisAgrostidetum capillaris Billy 2000]

Especies diferenciales: Serapias lingua L., Orchis morio L., O. ustulata L., O. laxiflora Lam., Carex caryophyllea Latourr. (Mercadal, 2019c: Tabla 119).

Ecología: prados mesófilos poco intervenidos.

Sintaxonomía: subasociación próxima florísticamente al Orchido-Serapiadetum linguae. 


\section{Conclusiones}

La revisión sintaxonómica de los prados mesófilos basales y de media montaña del orden Arrhenatheretalia elatioris en la Europa occidental realizada en la tesis doctoral del autor, nos ha permitido distinguir claramente tres alianzas: Arrhenatherion elatioris, típica del centro de Europa, Salvio-Dactylidion, endémica de la península itálica, y Brachypodio-Centaurenion, propia del sur de Europa (península ibérica y mediodía francés) y motivo del presente estudio.

Los resultados obtenidos también nos han permitido reorganización el esquema sintaxonómico clásico de los Arrhenatheretalia del suroeste de Europa y asignar las asociaciones adscritas tradicionalmente a la alianza Cynosurion cristati al Brachypodio-Centaureion, así como algunas comunidades ibéricas asignadas habitualmente a la alianza Arrhenatherion. Este cambio se ha debido al constatar que el Brachypodio-Centaureion representa una alianza vicariante, en el sudoeste europeo, de la alianza Cynosurion cristati centroeuropea. Así, las especies diagnósticas de la alianza BrachypodioCentaureion en el área de estudio son: Agrostis capillaris, Danthonia decumbens, Potentilla erecta, Carex ovalis, Hieracium pilosella, Myosotis discolor, Carum verticillatum, Centaurea nigra, Senecio jacobaea, Avenula marginata, Nardus stricta, Chamaemelum nobile, Lepidium heterophyllum, Colchicum montanum, Hypochaeris radicata y Prunella vulgaris. En cambio, el Cynosurion cristati de la Europa central se distingue por un conjunto de taxones raros 0 ausentes en el BrachypodioCentaureion: Ranunculus repens, Agrostis stolonifera, Phleum pratense, Plantago major, Lolium perenne, Cardamine pratensis, Scorzonera autumnalis, Alopecurus pratensis subsp. pratensis, Bellis perennis, Poa trivialis, P. annua, Carex nigra, Carex disticha y Potentilla anserina.

Dentro del Brachypodio-Centaureion incluimos, de momento, a 14 asociaciones (Agrostio-Cynosuretum, Caro-Cynosuretum, Lino-Cynosuretum, ColchicoCynosuretum nom. corr., Campanulo-Cynosuretum nom. inept., Agrostio-Arrhenatheretum, AnthemidoCynosuretum, Festuco-Cynosuretum, OrchidoSerapiadetum, Armerio-Arrhenatheretum, PhleoCynosuretum, Cypero-Cynosuretum nova, Pediculari-Galietum stat. novus, ScorzoneroAgrostidetum nova), de las cuales caracterizamos a cinco por ser nuevas o desarrollarse en Cataluña, el área central del estudio:

- Ass. Campanulo-Cynosuretum Nègre 1969 nom. inept.: pastos, a veces segados, del Pirineo y de las cordilleras litorales catalano-valencianas, de (300)800-1.500(1.700) m de altitud. Esp. diagnósticas: Agrostis capillaris, Festuca rubra, Achillea millefolium, Cerastium fontanum, Prunella vulgaris, Alchemilla hybrida, Phleum pratense subsp. pratense, Pimpinella saxifraga, Rhinanthus pumilus, Stachys officinalis. No corregimos el nombre de la asociación pues aún no sabemos a qué taxon corresponde la entidad Campanula lanceolata var. subrhomboidalis.

- Ass. Phleo-Cynosuretum Rodríguez-Rojo et Fernández-González 2014.: pastos, a veces segados, del sistema lbérico y de las cordilleras litorales del sur de Cataluña y del norte del País Valenciano. Esp. diagnósticas: Phleum pratense subsp. nodosum, Bellis perennis, Festuca trichophylla, Hieracium pilosella, Agrostis capillaris subsp. castellana, Trifolium striatum, Deschampsia cespitosa.

- Ass. Cypero-Cynosuretum Mercadal ass. nova: pastos húmedos de Cap de Creus (Cataluña), sobre suelos débilmente salobres, de 50-125 m de altitud. Esp. diagnósticas: Festuca arundinacea, Cyperus longus, Plantago crassifolia., Vicia sativa subsp. nigra, Trifolium squamosum, Oenanthe lachenalii.

- Ass. Pediculari-Galietum (M. Mayor et al. 1975) Mercadal stat. novus: prados de siega habitualmente pastados sobre terrenos oligrotróficos de reacción ácida o neutra del sector oriental del sistema Central Ibérico. Esp. diagnósticas: Armeria arenaria subsp. segoviensis, Dianthus deltoides, Pedicularis schizocalyx, Klasea nudicaulis, Erodium carvifolium, Juncus squarrosus, Alopecurus pratensis subsp. arundinaceus.

- Ass. Scorzonero-Agrostidetum Billy ex Mercadal ass. nova: prados de siega mesohigrófilos, frescos y acidófilos del macizo Septentrional occitano, situados entre los 350 y los $840 \mathrm{~m}$ de altitud. Esp. caract. y dif.: Scorzonera humilis, Carex panicea, Dactylorhiza maculata, Carum verticillatum, Myosotis nemorosa, Cardamine pratensis.

\section{Esquema sintaxonómico}

Los sintaxones con código numérico y en negrita son los caracterizados geobotánicamente; el resto, solo son mencionados en el texto.

Class. MOLINIO CAERULEAE-ARRHENATHERETEA ELATIORIS TX. 1937

Ord. ARRHENATHERETALIA ELATIORIS Tx. 1931

All. Brachypodio pinnati-Centaureion nemoralis $\mathrm{Br}$.Bl. 1967 [\#1]

Ass. Agrostio castellanae-Cynosuretum cristati Teles 1957

Ass. Caro verticillati-Cynosuretum cristati Bellot et Casaseca ex Tx. in Tx. et Oberd. 1958

Ass. Lino angustifolii-Cynosuretum cristati Allorge ex Oberd. et Tx. in Tx. et Oberd. 1958 [incl. GaudinioFestucetum pratensis Br.-BI. 1967]

Ass. Colchico montani-Cynosuretum cristati Oberd. et Tx. in Tx. et Oberd. 1958 nom. corr. [= Merendero pyrenaicae-Cynosuretum cristati Oberd. et Tx. in Tx. et Oberd. 1958 nom. inept.]

Ass. Campanulo subrhomboidalis-Cynosuretum cristati Nègre 1969 nom. inept. [\#1.1]

Ass. Agrostio castellanae-Arrhenatheretum bulbosi Teles 1970

Ass. Anthemido nobilis-Cynosuretum cristati Teles 1970 
Ass. Festuco amplae-Cynosuretum cristati RivasMartínez ex Fuente 1986

Ass. Orchido morionis-Serapiadetum linguae de Foucault 1986

Ass. Armerio segoviensis-Arrhenatheretum bulbosi Rodríguez-Rojo et Sánchez Mata 2006

Ass. Phleo nodosi-Cynosuretum cristati RodríguezRojo et Fernández-González 2014 [\#1.2]

Ass. Cypero Iongi-Cynosuretum cristati Mercadal nova [\#1.3]

Ass. Pediculari schizocalycis-Galietum veri (M. Mayor, T. E. Díaz, F. Navarro, F. Martínez et G. Andrés 1975) Mercadal stat. novus [\#1.4]

Ass. Scorzonero humilis-Agrostidetum capillaris Billy ex Mercadal nova [\#1.5]

subass. typicum [\#1.5.1]

subass. serapiadetosum linguae Billy ex Mercadal nova [\#1.5.2]

\section{Otros sintaxones citados en el texto}

Arrhenatherion elatioris Koch 1926; Calthion palustris Tx. 1937; Cynosuro cristati-Trifolietum repentis CortiniPedrotti, Orsomando et Pedrotti 1973; Juncion acutiflori Br.-Bl. in Br.-Bl. et Tx. 1952; Salvio pratensis-Dactylidion glomeratae Ubaldi, Zanotti et Corticelli in Ubaldi 2003 [= Ranunculo neapolitani-Arrhenatherion elatioris Allegrezza et Biondi 2011].

\section{Agradecimientos}

Al Dr. Jean-Marc Tison, de la Société Botanique de France, y al Dr. Llorenç Sáez, de la Universitat Autònoma de Barcelona, por los comentarios sobre distintos aspectos morfológicos de Campanula subsect. Heterophylla (Wit.) Fed.; al Dr. Luis Villar, investigador jubilado del Instituto Pirenaico de Ecología, por los datos bibliográficos facilitados; al Dr. Enrique Rico, de la Universidad de Salamanca, por los comentarios taxonómicos sobre los géneros Colchicum y Merendera; y a la Dra. Isabelle Chanaron, del Muséum d'histoire naturelle d'Aix-en-Provence, por la búsqueda incesante pero infructuosa del tipo de Campanula lanceolata var. subrhomboidalis.

\section{Bibliografía}

Aedo, C. \& Herrero, A. (2005). Orchidaceae. In S. Castroviejo, S. (Ed.), Flora iberica: plantas vasculares de la Península Ibérica e Islas Baleares, vol XXI. Real Jardín Botánico-CSIC. Madrid.

Aizpuru, I., Aseginolaza, C., Uribe-Echebarría, P., Urrutia, P. \& Zorrakin, I. (1999). Claves ilustradas de la flora del País Vasco y territorios limítrofes. Eusko Jaularitzaren Argitalpen Zerbitzu Nagusia. Gasteiz. 831 pp.

Álvarez de la Campa, J. M. (2004). Vegetació del massís del Port. Institut d'Estudis llerdencs. Lleida. 458 pp.

Arnelas, I. \& Devesa, J. A. (2011). Revisión taxonómica de Centaruea sect. Jacea (Mill.) Pers.
(Asteraceae) en la Península Ibérica. Acta Botanica Malacitana, 36: 33-88.

Bateman, R., Pridgeon, A. M. \& Chase, M. W. (1997). Phylogenetic of subtribe Orchidinae (Orchidoideae, Orchidaceae) based on nuclear ITS sequences. 2. Infrageneric relationships and reclassification to achieve monophyly of Orchis. Lindleyana, 12: 113-141

Billy, F. (2000). Prairies et pâturages en Basse Auvergne. Bulletin Société Botanique du CentreOuest, Nouvelle série-Número speciaux, 20, 1258.

Bolòs, O. de. (1967). Comunidades vegetales de las comarcas próximas al litoral situadas entre los ríos Llobregat y Segura. Memorias de la Real Academia de Ciencias y Artes de Barcelona, 38(1), 1-279.

Bolòs, O. de., Vigo, J., Masalles, R. M. \& Ninot, J. M. (2005). Flora manual dels Països Catalans. (3ed.). Pòrtic. Barcelona. 1310 pp.

Braun-Blanquet, J. (1964). Pflanzensoziologie. Grundzüge der vegetationskunde. (3ed.). Springer-Verlag. Wien. 865 pp.

Braun-Blanquet, J. (1967). Vegetationsskizzen aus dem Baskenland mit Ausblicken auf des Weitere Ibero-Atlantikum. II. Teil. Vegetatio, 14(1-4), 1126.

Braun-Blanquet, J. (1979). Fitosociología. Bases para el estudio de las comunidades vegetales. Blume. Madrid. 820 pp.

Castroviejo, S. (1986-2021). Flora iberica: plantas vasculares de la Península Ibérica e Islas Baleares. Real Jardín Botánico-CSIC. Madrid. http://www.floraiberica.es/ [12/2/2021].

Delforge, P. (2009). Orchis et monophylie. Les Naturalistes belges, 90(hors-série - spécial Orchidées $\left.n^{\circ} 22\right)$, 15-35.

Fennane, M., Ibn Tattou, M. \& El Oualidi, J. (Ed.). (2014). Flore pratique du Maroc, III. Institut Scientifique. Rabat. 793 pp.

Foucault, B. de. (2016). Contribution au prodome des végétations de France: les Arrhenatheretea elatioris Braun-Blanq. ex Braun-Blanq., Roussine \& Nègre 1952. Documents phytosociologiques, 3, 5-217.

Franquesa, T. (1995). El paisatge vegetal de la península del cap de Creus. Arxius de les Seccions de Ciències, CIX. Secció de Ciències Biològiques. IEC. Barcelona. 628 pp.

Gardou, C. (1972). Recherches biosystématiques sur la Section Jacea Cass. et quelques sections voisines du genre Centaurea L. en France et dans les régions limitrophes. Feddes Repertorium, 83(5-6), 311-472.

GBIF. (2021). Global Biodiversity Information Facility. https://www.gbif.org. [12/02/2021].

Géhu, J. M. (1999). Synsystématique des prairies de France. Annali di Botanica, LVII, 15-30. doi: https://doi.org/10.4462/annbotrm-9044.

Géhu, J. M. (2006). Dictionnaire de Sociologie et Synécologie végétales. J. Cramer. Stuttgart. 899 pp. 
Geslot, A. (1982). Les Campanules de la sous-section Heterophylla dans les Pyrénées: une étude de Biosystématique. Tesis doctoral inédita. AixMarseille-III.

Geslot, A., Villar, L. \& Palma, B. (1990). Chorologie des Campanules pyrénéennes de la sous-section Heterophylla (Wit.) Fed. Monografías del Instituto Pirenaico de Ecología, 5, 137-159.

Kaplan, Z., Danihelka, J., Chrtek, J., Krischner, J., Kubát, K., Stech, M. \& Stepanek, J. (Ed.). (2019). Klíc ke kvetene Ceské republiky. Academia. Praha. $1.168 \mathrm{pp}$.

López, G. (2021). Brachypodium P. Beauv. [borrador]. In S. Castroviejo (Ed.), Flora iberica: plantas vasculares de la Península Ibérica e Islas Baleares. Real Jardín Botánico-CSIC. Madrid.

Mayor, M., Díaz, T. E., Navarro, F., Martínez, G. \& Andrés, J. (1975). Los pastizales del Sistema Central. Nota I: Somosierra, Ayllón y Pela. Revista de la Facultad de Ciencias de la Universidad de Oviedo, 15-16: 238-322.

Mercadal, G. (2019a). Els prats de dall de la terra baixa catalana. Caracterització geobotànica, valoració agroambiental $i$ estudi de les relacions fitosociològiques entre els prats dalladors de l'Europa occidental. Volum 1: introducció $i$ cartografia de les unitats pradenques. Tesis doctoral. Universitat de Girona. 669 pp. doi: https://doi.org/10.13140/RG.2.2.24042.75200.

Mercadal, G. (2019b). Els prats de dall de la terra baixa catalana. Caracterització geobotànica, valoració agroambiental i estudi de les relacions fitosociològiques entre els prats dalladors de I'Europa occidental. Volum 2: flora. Tesis doctoral. Universitat de Girona. 881 pp. doi: https://doi.org/10.13140/RG.2.2.21460.63365.

Mercadal, G. (2019c). Els prats de dall de la terra baixa catalana. Caracterització geobotànica, valoració agroambiental i estudi de les relacions fitosociològiques entre els prats dalladors de l'Europa occidental. Volum 3: vegetació i gestió pradenca. Tesis doctoral. Universitat de Girona. $756+5$ pp.

https://doi.org/10.13140/RG.2.2.31802.21441.

Mercadal, G. (2020a). Caracterització geobotànica dels prats de dall mesòfils de l'associació Odontito serotini-Trifolietum pratensis 0 . Bolòs et Masalles 1983 (all. Arrhenatherion elatioris Koch 1926) dels Pirineus orientals catalans. In J. Bou \& L. Vilar (Eds.), Actes del XII Col-loqui Internacional de Botànica Pirenaica-Cantàbrica (pp. 181-234). Documenta universitaria. Disponible en https://www.researchgate.net/publication/342130 $153[12 / 06 / 2020]$.

Mercadal, G. (2020b). Caracterització geobotànica i validació fitosociològica de diversos sintàxons de prats de dall higròfils (ord. Deschampsietalia cespitosae) i mesohigròfils (ord. TrifolioHordeetalia) de Catalunya i de l'Europa occidental. Butlletí de la Institució Catalana d'Història Natural, 84: 163-193.

https://doi.org/10.2436/20.1502.01.49.
Mercadal, G. (2020c). Caracterización geobotánica y validación fitosociológica de algunos sintaxones de prados salobres (all. Juncion maritimi y Plantaginion crassifoliae, class. Juncetea maritimi) de Cataluña y del Mediterráneo occidental. Acta Botanica Malacitana, 45.

http://dx.doi.org/10.24310/abm.v45i0.9603.

Mercadal, G. (2020d). Caracterización geobotánica de algunos sintaxones de prados de siega (all. Arrhenatherion elatioris) de Cataluña y de la Europa occidental. Flora Montiberica, 78. 120133.

https://www.researchgate.net/publication/345177 510. [12/02/2021].

Mercadal, G. (2021). Geobotanical characterisation of the Trifolion squamosi nom. corr. alliance (class. Juncetea maritimi) in Catalonia and the Western Mediterranean. Hacquetia, 20(1), 107-117. doi: https://doi.org/10.2478/hacq-2020-0019.

Molero, J. (1976). Estudio florístico y fitogeográfico de la sierra de Montsant y su área de influencia. Tesis Doctoral inédita. Universitat de Barcelona.

Mucina, L., Bültmann, K., Dierßenm, K., Theurillat, J.-P., Raus, T., Čarni, A., Šumberová, K., Willner, W., Dengler, J., Gavilán, R., Chytrý, M., Hájek, M., Di Pietro, P., laukushenko, D., Pallas, J., Daniëls, F.J.A., Bergmeier, E., Santos Guerra, A., Ermakov, N., Valachovič, M.; Schaminée, J.H.J., Lysenko, T., Diduhk, Y.P., Pignatti, S., Rodwell, J.S., Capelo, J., Weber, H.E., Solomeshch, A., Dimopoulus, P., Aguiar, C., Hennekens, S.M. \& Tichý, L. (2016). Vegetation of Europe: hierarchical floristic classification system of vascular plant, bryophyte, lichen, and algal communities. Applied Vegetation Science 19(1), 3-264. https://doi.org/10.1111/avsc.12257.

Nègre, E. (1969). La végétation du bassin de l'One (Pyrénées Centrales). Deuxième note: les Pelouses. Portugaliae Acta Biologica (B), 10, 1-135.

Ozenda, P. (1994). La végétation du continent européen. Delachaux et Niestlé. Lausanne. 271 pp.

Parolly, G. \& Rohwer, J. G. (2019). Schmeil-Fitschen. Die Flora Deutschlands und angrenzender Länder. (97ed.). Quelle \& Meyer. Wiebelsheim. 980 pp.

Pignatti, S., Guatino, R. \& La Rosa, M. (2017). Flora d'Italia, vol 1. (2ed.). Edagricole. Milano. 1.064 pp.

Reymann, J., Panaïotis, C., Bioret, F., Bacchetta, G., Delage, A.; Delbosc, P., Gamisans, J., Gauberville, C., Hugot, L., O'deye-Guizien, K., Piazza, C. \& Pioli, A. (2016). Prodome des végétations de Corse. Documents phytosociologiques, 4, 1-175.

Rico, E. (2013a). Merendera Ramond. In S. Castroviejo, S. (Ed.), Flora iberica: plantas vasculares de la Península Ibérica e Islas Baleares, vol XX. Real Jardín Botánico-CSIC. Madrid.

Rico, E. (2013b). Colchicum L. In S. Castroviejo, S. (Ed.), Flora iberica: plantas vasculares de la Península lbérica e Islas Baleares, vol XX. Real Jardín Botánico-CSIC. Madrid.

Rivas Goday, S. \& Rivas-Martínez, S. (1963). Estudio y clasificación de los pastizales españoles. Vol. 
127. Publicaciones del Ministerio de Agricultura. Madrid. 269 pp.

Rivas-Martínez, S., Díaz, T. E., Penas, A. \& Fernández, F. (2011). Mapa de series, geoseries y geopermaseries de vegetación de España. Memoria del mapa de Vegetación de España, parte II. Itinera Geobotanica, 18(1), 5-424. https://floramontiberica.files.wordpress.com/2013/ 01/itinerageobotanica_181_2011.pdf. [12/02/2021].

Rivas-Martínez, S., Fernández-González, F., Loidi, J., Lousa, M. \& Penas, A. (2001). Syntaxonomical checklist of vascular plant communities of Spain and Portugal to association level. Itinera Geobotanica, 14, 5-341. Disponible en https://www.researchgate.net/publication/216034 $253[12 / 06 / 2020]$.

Rodríguez-Rojo, M. P. \& Fernández González, M. (2014). Diversity Patterns and Typology of Cynosurus cristatus Grasslands (Cynosurion cristati Tüxen 1947) in the Iberian Peninsula. Folia Geobotanica, 49, 461-485.

Rodríguez-Rojo, M. P., Jiménez-Alfaro, B., Jandt, U., Bruelheide, H., Rodwell, J. S., Schaminée, J. H. J., Perrin, P. M., Zygmunt, K., Wilner, W., FernándezGonzález, F. \& Chytry, M. (2017). Diversity of lowland hay meadows and pastures in Western and Central Europe. Applied Vegetation Science, 20, 702-719. https://doi.org/10.1111/avsc.12326.

Sáez, L. \& J. J. Aldasoro. (2001). Campanula L. In S. Castroviejo, S. (Ed.), Flora iberica: plantas vasculares de la Península Ibérica e Islas
Baleares, vol XIV. Real Jardín Botánico-CSIC. Madrid.

Schippmann, U. (1991). Revision der europäischen Arten der Gattung Brachypodium Palisot de Bauvois (Poaceae). Boissiera, 45, 1-250.

Theurillat, J-P., Willner, W., Fernández-González, F., Bültmann, K., Čarni, A., Gigante, D., Mucina, L. \& Weber, H. E. (2020). International Code of Phytosociological Nomenclature. 4th edition. Applied Vegetation Science.

https://doi.org/10.1111/avsc. 12491.

Tison, J.-M. \& Foucault, B. de. (2014). Flora Gallica. Biotope. Mèze. $1196 \mathrm{pp}$.

Tyteca, D. \& Klein, E. (2008). Genes, morphology and biology - The systematics of Orchidinae revisited. Journal Europäischer Orchideen, 40, 501-544.

Villegas, N. (1993). Flora $i$ vegetació de les muntanyes del Puigsacalm-Serra de Milany. Tesis doctoral inédita. Universitat de Barcelona.

Villegas, N. (1997). Carici ornithopodae-Agrostidetum capillaris ass. nova, un tipus de falguerar de la muntanya mitjana humida. Estudi fitocenològic. Butlletí de la Institució Catalana d'Història Natural, 65: 31-42.

WCVP. (2021). World Checklist of Vascular Plants. Royal Botanic Gardens Kew. Disponible en https://wcvp.science.kew.org. [12/02/2021]

Weber, H. E., Moravec, J. \& Theurillat, J.-P. (2000). International Code of Phytosociological Nomenclature. 3rd edition. Journal of Vegetation Science, 11: 739-768. https://doi.org/10.2307/3236580. 